\title{
The use of Mycobacterium tuberculosis HspX and GlcB proteins to identify latent tuberculosis in rheumatoid arthritis patients
}

\author{
Bruna Daniella Souza Silva', Daniela Graner Schuwartz Tannus-Silva², \\ Marcelo Fouad Rabahi², Andre Kipnis' ${ }^{1}$ Ana Paula Junqueira-Kipnis¹/+ \\ 1Departamento de Microbiologia, Imunologia, Parasitologia e Patologia, Instituto de Patologia Tropical e Saúde Pública \\ ${ }^{2}$ Departamento de Clínica Médica, Faculdade de Medicina, Universidade Federal de Goiás, Goiânia, GO, Brasil
}

\begin{abstract}
Rheumatoid arthritis (RA) is an autoimmune disease characterised by the destruction of articular cartilage and bone damage. The chronic treatment of RA patients causes a higher susceptibility to infectious diseases such as tuberculosis (TB); one-third of the world's population is latently infected (LTBI) with Mycobacterium tuberculosis (Mtb). The tuberculin skin test is used to identify individuals LTBI, but many studies have shown that this test is not suitable for RA patients. The goal of this work was to test the specific cellular immune responses to the Mtb malate synthase (GlcB) and heat shock protein $X(H s p X)$ antigens of $R A$ patients and to correlate those responses with LTBI status. The T-helper (Th)1, Th17 and Treg-specific immune responses to the GlcB and HspX Mtb antigens were analysed in RA patients candidates for tumour necrosis factor- $\alpha$ blocker treatment. Our results demonstrated that LTBI RA patients had Th1-specific immune responses to GlcB and HspX. Patients were followed up over two years and $14.3 \%$ developed active TB. After the development of active TB, RA patients had increased numbers of Th17 and Treg cells, similar to TB patients. These results demonstrate that a GlcB and HspX antigen assay can be used as a diagnostic test to identify LTBI RA patients.
\end{abstract}

Key words: Th cells - anti-TNF- $\alpha$ - latent infected tuberculosis

Rheumatoid arthritis (RA) is an autoimmune disease of unknown aetiology that is characterised by chronic inflammation of the joints and the subsequent destruction of articular cartilage and bone damage (Mota et al. 2012). The pathogenesis of RA involves many factors, including interleukin (IL)-1, tumour necrosis factor (TNF)- $\alpha$ and IL-17 cytokines, macrophages and T and B cell populations (Brennan \& McInnes 2008, Scott et al. 2010). The involvement of many subtypes of T-helper (Th) cells has been demonstrated in the immune responses of RA patients (Cope 2008); therefore, these cells were predicted to play a crucial role in RA development. It is believed that the Th1 and Th17 cell subtypes are mainly responsible for the inflammatory responses in this disease (Chabaud et al. 1999, Cope 2008). Recent studies have indicated that imbalances of Th1/Th2 and Th17/Treg cells may be responsible for the development and progression of RA (Boissier et al. 2008, Wang et al. 2012).

An RA patient's therapy varies according to both the disease stage and its severity and activity. Current drug treatment consists of anti-inflammatory drugs and, if necessary, biological agents such as TNF- $\alpha$ blockers (Aaltonen et al. 2012). The continuous medical treatment

doi: 10.1590/0074-02760140140

Financial support: CNPq (301976/2011-2, 472909/2011-8, 479605/2007-6)

BDSS received a fellowship from CNPq (133655/2009-1).

+ Corresponding author: apkipnis@gmail.com

Received 8 March 2013

Accepted 3 October 2013 of RA patients renders them more susceptible to infectious diseases such as tuberculosis (TB) through exposure to Mycobacterium tuberculosis (Mtb) bacilli or TB reactivation (Jick et al. 2006).

Brazil is one of the 22 countries where $90 \%$ of all TB cases in the world are found (Barry et al. 2009, WHO 2011). One of the main ways to improve TB control is by predicting disease progression. Characterising the host immune response will aid in defining if one will naturally resolve the infection or develop latent or active TB. Although the specific immune response to Mtb has been extensively studied, it has not been clarified in patients with RA. Therefore, understanding the immune response to TB antigens in these patients will build knowledge and will contribute to the development of new diagnostic tests.

The tuberculin skin test (TST) identifies healthy individuals with latent tuberculosis infection (LTBI) (Nienhaus et al. 2013). This method is neither sensitive enough nor fully reliable, particularly in immunocompromised patients (Barry et al. 2009, Tannus-Silva et al. 2012). The recently developed interferon (IFN)- $\gamma$ release assays (IGRA) measure TB-specific T cells responding to Mtb-derived antigens, including early secreted antigenic target 6 (ESAT-6) and culture filtrate protein 10 (CFP-10). Currently, the IGRA is regarded as the first real advance in LTBI diagnostics.

The specific cellular immune responses to Mtb antigens have been extensively described (Babu et al. 2010, Rueda et al. 2010). Patients with active TB have Mtb-specific Th1, Th17 and Treg cells in the peripheral blood (PB) (Guyot-Revol et al. 2006, Hougardy et al. 2007, Araújo-Filho et al. 2008, Scriba et al. 2008, Cruz 
et al. 2009). Many antigens, such as heat shock protein $\mathrm{X}(\mathrm{HspX})$ antigens, can be used to analyse the overall immune responses of LTBI individuals (Tavares et al. 2006, Rabahi et al. 2007, Reis et al. 2009); however, these antigens have not been tested for specific cellular responses. Thus, the objectives of this work were to analyse the specific immune responses of Th1, Th17 and Treg cells from RA patients to malate synthase (GlcB) and HspX from Mtb and to evaluate the use of these responses for identifying LTBI RA patients.

\section{SUBJECTS, MATERIALS AND METHODS}

Participants - For this longitudinal, descriptive study, RA and TB patients were recruited at the Clinical Hospital and the Hospital for Tropical Diseases, from June 2009-June 2010. Forty-two of these individuals participated in the study and signed informed consent forms. During the same period, control subjects (CS) - individuals with no TB, no RA and no symptoms of other investigative disease - were recruited at Federal University of Goiás (UFGO); 23 of these individuals participated and provided informed consent. Sixty-five individuals participated in the study. RA and TB patients were recruited independently of sex and race. Inclusion criteria for RA patients were an RA diagnosis according to the American College of Rheumatology and no prior treatment with anti-TNF- $\alpha$ antibodies (Abs). Inclusion criteria for TB patients were pulmonary TB diagnosis based on bacilloscopy, microbiological cultures and clinical and radiological features within 15 days of TB treatment. Exclusion criteria for all individuals were pregnancy, positive serology for human immunodeficiency virus and age (younger than 18 years). The procedures followed the Helsinki Declaration for human experimentation.

$T S T$ - RA and CS ( $\mathrm{n}=21$ for RA and $\mathrm{n}=23$ for CS) were given the TST to characterise LTBI. Patients received $0.1 \mathrm{~mL}$ of purified protein derivative (PPD-RT 23 Mtb-2 UT, Copenhagen, Denmark) intradermally (Mantoux technique) in the left forearm. For the CS, the size of skin induration was measured along the longer transverse axis $72 \mathrm{~h}$ later and the result was expressed in millimetres. Individuals with TST $\leq 9 \mathrm{~mm}$ were classified as TST- and were CS $(\mathrm{n}=12)$. TST $>10 \mathrm{~mm}$ were classified as TST+ and were CS-LTBI+ $(\mathrm{n}=11)$; RA individuals with TST $\leq 4 \mathrm{~mm}$ were classified as TSTand were LTBI- $(\mathrm{n}=11)$. TST $>5 \mathrm{~mm}$ were classified as TST + and were LTBI+ $(\mathrm{n}=10)$ according to the World Health Organization (WHO 2003).

PB mononuclear cells (PBMCs) and cell culture - PBMCs were obtained from all participants by first collecting whole blood using heparin as an anticoagulant, followed by Ficoll density gradient centrifugation (Ficoll-Paque Plus, GE Healthcare Bio-Sciences AB). The cells were washed twice in saline and distributed in 96-well plates at $2 \times 10^{5}$ cells $/ \mathrm{mL}$ in RPMI-1640 medium (GIBCO, Invitrogen Corporation) supplemented with 2 $\mathrm{mM}$ glutamine, $10 \mathrm{nM}$ pyruvate, $2 \mathrm{mM}$ amino acids, 50 $\mu \mathrm{g} / \mathrm{mL}$ penicillin, $50 \mu \mathrm{g} / \mathrm{mL}$ streptomycin and $10 \%$ heatinactivated bovine serum. The cells were then incubated with recombinant $\mathrm{Mtb}$ antigens $(\mathrm{rGlcB}$ and $\mathrm{rHspX}, 1$ $\mu \mathrm{g} / \mathrm{mL}$ ), produced by our group as previously reported (Araújo-Filho et al. 2008), or with phytohaemagglutinin (PHA) $(1 \mu \mathrm{g} / \mathrm{mL})$ as a positive control and were cultivated at $37^{\circ} \mathrm{C}$ with $5 \% \mathrm{CO}_{2}$ for $96 \mathrm{~h}$ in the presence of anti-CD3 (eBioscience). Cells stimulated with medium alone or with anti-CD3 were used as controls.

Flow cytometry - The following Abs were used for surface and intracellular staining for flow cytometry: IFN- $\gamma$-fluorescein isothiocyanate (FITC) (BD Bioscience Pharmingen), CD25-PerCPCy5.5, IL-10-PE, forkhead box protein 3 (FOXP3)-FITC, CD4-APC, T-bet-PE, streptavidin-FITC,IL-17-PE(eBioscience), IL-23R-Biotin (R\&D Systems) and transforming growth factor (TGF)$\beta$-PE (IQ Products). Cells were labelled with CD4-APC and with rat IgG1-PE isotype control to set the gate for IL-17, IL-10 and TGF- $\beta$-positive cells. Cells were also labelled with CD4-APC and with rat IgG1-FITC isotype control to set the gate for IFN- $\gamma$-positive cells. For flow cytometry analysis, cells stimulated with medium alone, PHA or TB antigen were treated with Golgi Stop Solution (containing monensin, BD Biosciences Pharmingen) and after 4-6 h of further incubation, the cells were harvested for analysis. The cells were treated with phosphate buffered saline (PBS) containing $0.05 \%$ azide for $20 \mathrm{~min}$ prior to surface and intracellular staining. After centrifugation (3,000 rpm for $10 \mathrm{~min}$ ), cells were stained at $4^{\circ} \mathrm{C}$ for 18 min with surface marker Abs [CD4-APC and CD25-PerCPCy5.5 (eBioscience) and IL-23R-Biotin (R\&D Systems)]. The plates were then treated with streptavidin-FITC for $18 \mathrm{~min}$ in the dark. Subsequently, the plates were washed twice with PBS containing $0.05 \%$ azide and treated with PermFix (BD Pharmingen, San Jose, CA, USA) for $18 \mathrm{~min}$. For intracellular staining, cells were permeabilised with Perm Wash buffer (BD Biosciences Pharmingen) and incubated at $4^{\circ} \mathrm{C}$ for $18 \mathrm{~min}$ with the following specific Abs: IFN- $\gamma$-FITC (BD Biosciences Pharmingen), IL-10-PE, FOXP3-FITC, T-betPE, IL-17-PE (eBioscience) and TGF- $\beta$-PE (IQ Products). To detect FOXP3, cells were treated using the FOXP3 Staining Buffer Set (eBioscience). After washing, the samples were immediately analysed on a FACSCalibur flow cytometer (Becton \& Dickinson, San Jose, CA, USA) at the Araújo Jorge Hospital (state of Goiás, Brazil). At least 100,000 events were acquired per sample. Data analysis was performed using FACSDiva software (BD Biosciences, Becton \& Dickinson).

IL- 6 detection - Levels of IL- 6 in the plasma were measured using the standard Human IL-6 ELISA ReadySET-Go! Kit (eBioscience) according to the manufacturer's instructions. The concentrations of IL- 6 in the samples were calculated using a standard curve generated from recombinant IL-6 and the results were expressed as $\mathrm{pg} / \mathrm{mL}$. The sensitivity of the assay was $2 \mathrm{pg} / \mathrm{mL}$.

Statistical analysis - Means and standard errors of the mean were calculated. After assessing the normality of all data by quantile plots, one-way ANOVA was used to compare variances between the groups. Because the variance magnitude of each group was not vastly different, an F-test and a p-value were calculated to predict the 
differences between the averages of each group. A posthoc test (Student $t$ test or Mann Whitney $U$-Wilcoxon) was used to evaluate if each specific group pair was different from each other. A $p$ value less than $<0.05$ was considered to be statistically significant.

Ethics - All studies were approved by the Hospital of the UFGO Ethical Committee (protocol 195/07, $055 / 2009$ ) and informed consent was obtained from all participating subjects. The procedures followed the Helsinki's Declaration for human experimentation.

\section{RESULTS}

To evaluate the immune responses to GlcB and HspX, blood from RA patients, TB patients and CS was analysed. The RA patient clinical parameters used in this study are presented in Table I. Twenty-one patients with RA (average age $=55.6$ years $)$ classified as either LTBI $+(n=10)$ or LTBI- $(\mathrm{n}=11)$ were characterised according to their clinical statuses, treatment, BCG vaccination and concomitant disease. RA individuals were diagnosed between 10-35 years prior to enrolment and the majority of them were BCG-vaccinated and were receiving anti-inflammatory treatment for RA. Hypertension was the predominant concomitant disease. As expected, $70 \%$ of the RA LTBI+ individuals indicated previous contact with active TB patients. All RA patients were followed for two years and $14.3 \%(n=3)$ developed active TB during this period (TBRA). All three individuals, whose average age was 48.3 years, had previous contact with active TB patients.

The characteristics of TB patients and control individuals are shown in Table II. Recently diagnosed pulmonary TB patients $(n=21)$ with an average age of 41.9 years were mainly male $(61.9 \%)$; the majority of them were BCG vaccinated and only $42 \%$ indicated previous contact with patients with active TB. The control group consisted of individuals with an average age of 38 years and they were further stratified as LTBI- $(\mathrm{n}=12)$ or LTBI+ $(\mathrm{n}=11)$. The majority of the control individuals had been vaccinated with BCG prior to six months of age (Table II).

The existence of different $\mathrm{T}$ cell subsets determines $\mathrm{T}$ cell responses and the host immune status. Many $\mathrm{T}$ cell subtypes are involved in the immune response to TB, independent of associated disease. We investigated the frequencies of $\mathrm{T}$ cell subsets in patients with RA and their correlation to Mtb infection by determining specific cellular function and cytokine production in these patients. To accomplish this analysis, patients with RA were classified as LTBI+ or non-LTBI- according to TST.

TABLE I

Clinical characteristics of rheumatoid arthritis (RA) patients according to tuberculin skin test (TST) statuses

\begin{tabular}{|c|c|c|}
\hline & $\begin{array}{l}\text { RA LTBI- } \\
(\mathrm{n}=11) \\
\mathrm{n}(\%)\end{array}$ & $\begin{array}{l}\text { RA LTBI+ } \\
\quad(\mathrm{n}=10) \\
\mathrm{n}(\%)\end{array}$ \\
\hline Mean age (min-max) (years) & $59(21-75)$ & $52(37-63)$ \\
\hline Woman & $9(81.8)$ & $10(100)$ \\
\hline Mean time since diagnosis (min-max) (years) & $13.2(2-35)$ & $7.8(2-17)$ \\
\hline BCG & $7(63.6)$ & $7(70)$ \\
\hline History of contact with tuberculosis & $3(27.7)$ & $7(70)$ \\
\hline Current smokers and ex-smokers & $5(45.4)$ & $4(40)$ \\
\hline Use of corticosteroids (prednisone or equivalent) & $11(100)$ & $10(100)$ \\
\hline $\begin{array}{l}\text { Use of a disease modifying anti-rheumatic drug } \\
\text { (methotrexate or azathioprine or leflunomide) }\end{array}$ & $8(72.7)$ & $9(90)$ \\
\hline $\begin{array}{l}\text { Use of corticosteroids (prednisone or equivalent) } \\
\text { and disease modifying anti-rheumatic drugs } \\
\text { (methotrexate or azathioprine or leflunomide) }\end{array}$ & $7(63.6)$ & $8(80)$ \\
\hline \multicolumn{3}{|l|}{ Concomitant diseases } \\
\hline Hypertension & $7(63.6)$ & $2(20)$ \\
\hline Diabetes mellitus & $2(18.1)$ & $0(0)$ \\
\hline Bone disorders & $3(27.7)$ & $0(0)$ \\
\hline Cancer & $2(18.1)$ & $0(0)$ \\
\hline Hepatitis & $2(18.1)$ & $1(10)$ \\
\hline Asthma & $0(0)$ & $1(10)$ \\
\hline Hypothyroidism & $3(27.7)$ & $1(10)$ \\
\hline Dyslipidemia & $1(9.1)$ & $0(0)$ \\
\hline Angina & $1(9.1)$ & $0(0)$ \\
\hline Glaucoma & $0(0)$ & $1(10)$ \\
\hline Leprosy & $1(9.1)$ & $0(0)$ \\
\hline
\end{tabular}

LTBI: latent infected tuberculosis. 
Once it was well established that Th1-specific cells are important for eliminating mycobacteria during active $\mathrm{TB}$, we asked if LTBI infection induces specific immune responses and the expansion of Th1-specific cells against rGlcB and $\mathrm{rHspX}$ antigens in RA patients. Th1 cells were characterised as $\mathrm{CD} 4^{+} \mathrm{T}$-bet ${ }^{+} \mathrm{IFN}-\gamma^{+}$by flow cytometry (Fig. 1A). We observed that RA LTBI+ patients had a specific Th1 response to Mtb antigens (Fig. 1B, C) $(p<0.05)$, similar to CS-LTBI+ individuals and TB patients. These results demonstrate that LTBI in RA patients induces the proliferation of Th1 cells specific to $\mathrm{rGlcB}$ and $\mathrm{rHspX}$.

It is known that Th17 cells are correlated with TB infection. Although Th17 cell levels in synovial fluid and blood are elevated among RA patients, their specificity is not known. Therefore, we evaluated whether these cells could specifically respond to Mtb antigens. Th17 cells are characterised by the expression of the IL-23 receptor on their cell surfaces and by IL-17 production (Mus et al. 2010) (Fig. 2A). As shown in Fig. 2B, C, RA patient Th17 cells did not recognise rGlcB or rHspX. Therefore, expansion of Th17-specific cells is induced only during active TB.

Th17 and Treg cells can be induced by IL- 6 and previous studies have reported that this cytokine was significantly elevated in patients with TB and RA (Okada et al. 2011, Wang et al. 2012, Zhang et al. 2012). IL-6 is involved in the pathogenesis of RA and contributes to tissue destruction. The levels of this cytokine in RA patients were measured in the plasma and were correlated with their LTBI statuses. RA LTBI+ individuals presented higher plasma levels of IL- 6 when compared with RA LTBI- individuals (Fig. 2D) $(\mathrm{p}<0.05)$.

Although patients with active TB have Th1 cells specific to Mtb antigens, this response alone is not sufficient to control the infection. Some authors believe that Treg cells are involved in the suppression of Mtb-specific Th1 cells (Chen et al. 2007, Roberts et al. 2007). Human CD4 ${ }^{+}$ Treg cells can be distinguished from recently activated $\mathrm{T}$ cells $\left(\mathrm{CD} 25^{+}\right)$by their expression of the nuclear transcription factor FOXP3 (Fontenot et al. 2003, Roberts et al. 2007, Ellner 2010). Treg cells have been shown to regulate effector T cells (Ribeiro-Rodrigues et al. 2006, Chen et al. 2007). Therefore, we asked whether Treg $\left(\mathrm{CD} 4{ }^{+} \mathrm{CD} 25^{+} \mathrm{FOXP} 3+\right)$ cells could be found in the PBMCs of RA patients. RA LTBI+ patients had lower levels of Treg cells, similar to CS and CS-LTBI+ individuals when compared to active TB patients (Fig. 3A) $(p<0.05)$.

Treg cells were analysed by flow cytometry for TGF- $\beta$ and IL-10 expression after stimulation with $\mathrm{rGlcB}$ or $\mathrm{rH}-$ spX. Fig. 3B shows that only active TB patients had TGF$\beta$-expressing Treg cells specific for $\mathrm{rGlcB}(\mathrm{p}<0.05)$, whereas TB and CS-LTBI+ individuals had TGF- $\beta+$ Treg cells specific to rHspX (Fig. 3C) $(\mathrm{p}<0.05)$. No differences were noted in IL-10 production by Treg cells from all patient groups and in response to both antigens (Fig. 3D, E). These results provide evidence that specific TGF- $\beta+$ Treg cells are only induced in active TB.

All RA patients were followed for a period of two years and $14.3 \%$ of them $(n=3)$ developed active TB. During this period, the drugs and dosages used to treat these patients were not altered. The patients who developed active TB were LTBI-positive. These individuals had increased levels of Th1, Th17 and Treg cells specific to both rGlcB (data not shown) and rHspX (Fig. 4A-E) $(\mathrm{p}<0.05)$. These responses were similar to the responses observed in TB patients. Interestingly, TB-RA patients had increased levels of specific IL-10+ Treg cells (Fig. 4E), which were not observed in patients with active TB (Fig. 3E).

TABLE II

Clinical characteristics of control subjects and tuberculosis (TB) patients

\begin{tabular}{lccc}
\hline & $\begin{array}{c}\mathrm{CS} \\
\mathrm{n}=12) \\
\mathrm{n}(\%)\end{array}$ & $\begin{array}{c}\text { CS-LTBI+ } \\
(\mathrm{n}=11) \\
\mathrm{n}(\%)\end{array}$ & $\begin{array}{c}\mathrm{TB}^{a} \\
(\mathrm{n}=21) \\
\mathrm{n}(\%)\end{array}$ \\
\hline Mean age (min-max) (years) & $37.2(18-66)$ & $39.1(21-68)$ & $41.9(21-71)$ \\
Woman & $4(33.3)$ & $6(54.6)$ & $8(38.1)$ \\
BCG & $12(100)$ & $9(81.8)$ & $14(66.6)$ \\
History of contact with TB & $2(16.6)$ & $6(54.5)$ & $9(42.8)$ \\
Concomitant diseases & & $3(27.2)$ & $4(19)$ \\
Hypertension & $2(16.6)$ & $1(9.1)$ & $1(4.7)$ \\
Diabetes mellitus & $1(8.3)$ & $1(9.1)$ & $2(9.5)$ \\
Anaemia & $1(8.3)$ & $0(0)$ & $2(9.5)$ \\
Gout & $0(0)$ & $0(0)$ & $1(4.7)$ \\
Pneumonia & $0(0)$ & $1(9.1)$ & $1(4.7)$ \\
Hypothyroidism & $0(0)$ & $0(0)$ & $1(4.7)$ \\
Angina & $0(0)$ & $0(0)$ & $0(0)$ \\
Asthma & $1(8.3)$ & & \\
\hline
\end{tabular}

$a$ : TB patients submitted to treatment with three drugs: rifampin, isoniazid, pyrazinamide; CS: control subjects; LTBI: latent infected tuberculosis. All TB patients completed their treatment after six months. 


\section{DISCUSSION}

In this study, we demonstrate for the first time that rGlcB or rHspX can be used to identify LTBI in RA patients. In addition, we have shown that certain candidates for the use of TNF- $\alpha$ blockers, such as RA individuals, with LTBI were at increased risk for developing active TB; during the two-year follow up period, since 14.3\% $(n=3)$ of those patients developed active TB.

GlcB and HspX are antigens that can be used to evaluate the specific immune responses to latent and active TB. Many studies demonstrated that individuals with active and LTBI have specific humoural and cellular immune responses to HspX (Rabahi et al. 2007, Rueda et al. 2010). The GlcB antigen was used to evaluate the cellular response in individuals with active and multidrug-resistant TB (Araújo-Filho et al. 2008). The results described here correlate with the data shown in the literature and confirm the importance of these antigens for evaluating the immune response to TB.

The specific cellular immune responses to TB antigens such as ESAT- 6 and CFP-10 antigens, which are present in the RD1 region of Mtb, were analysed by TST or IGRA in RA patients (Vassilopoulos et al. 2008). To our knowledge, this work is the first study to evaluate cell subtypes by their specific immune responses to Mtb antigens in the active (GlcB) and latent (HspX and GlcB) phases of TB. These antigens were specifically recognised by Abs and T cells from active and LTBI patients who had previously received BCG vaccinations in endemic areas and who were thus important for this study (Rabahi et al. 2007, Reis et al. 2009). Therefore, combining one antigen expressed by Mtb during the latent phase of infection with another more ubiquitous antigen may improve the diagnosis of LTBI individuals. Curiously, in this study, RA LTBI+ patients had specific cellular immune responses to GlcB and HspX, similar to CS-LTBI+ controls and TB patients, demonstrating the importance of these antigens for the development of a new cell-based diagnostic test for TB.

Diagnosis of LTBI in RA patients is a challenging task. Due to the chronic nature of RA, treatment with anti-inflammatory drugs occasionally fails. In these cases, the patients need to be treated using biological drugs such as anti-TNF- $\alpha$. It is well known that TNF- $\alpha$ is a crucial cytokine for protection against TB (Barry et al. 2009, Ellner 2010) and, in its absence, latent individuals develop active TB. These facts highlight the importance of the development of an excellent test to screen RA patients who are candidates for drugs targeting the immune system. The candidate TB diagnostic tests to replace the
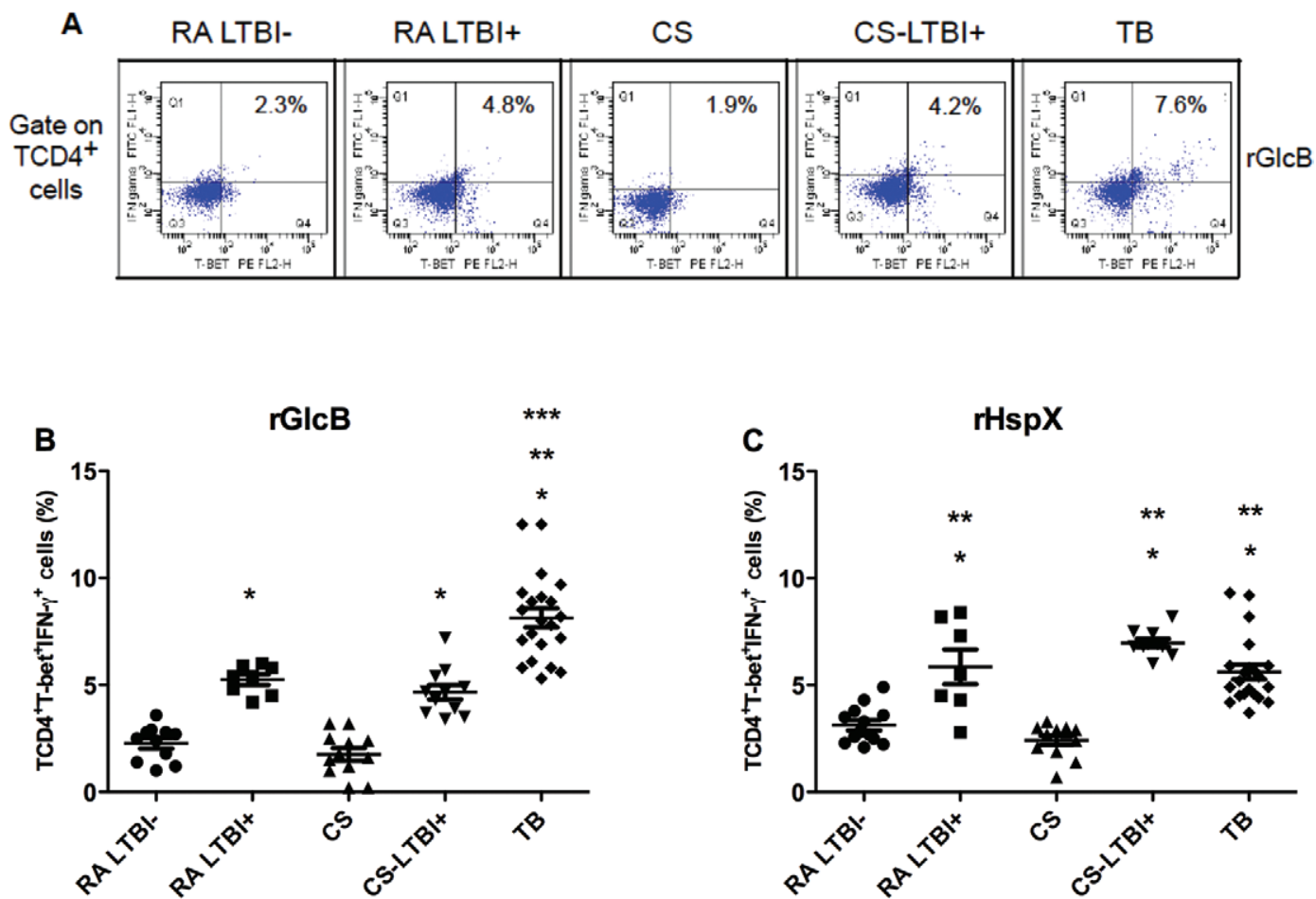

Fig. 1: specific T-helper (Th)1 [CD4 ${ }^{+}$T-bet $^{+}$interferon (IFN)- $\left.\gamma^{+}\right]$immune responses to recombinant malate synthase (rGlcB) or recombinant heat shock protein $\mathrm{X}(\mathrm{rHspX})$ are higher in control subjects with latent tuberculosis infection (CS-LTBI+) individuals, TB and rheumatoid arthritis (RA) LTBI+ patients $(\mathrm{p}<0.05)$. Peripheral blood mononuclear cells isolated from blood of CS $(\mathrm{CS}=\mathrm{TST}-$ and CS-LTBI+ $=$ TST +$)$, TB and RA (LTBI+ and LTBI-) patients were analysed for surface expression of CD4 and intracellular expression of T-bet and IFN- $\gamma$ by flow cytometry. A: dot plots representing specific Th1 immune response in RA LTBI-, RA LTBI+, CS, CS-LTBI+ individuals and TB patients; B: percentage of Th1 cells among RA LTBI- $(\mathrm{n}=11)$, RA LTBI+ $(\mathrm{n}=10), \mathrm{CS}(\mathrm{n}=12)$, CS-LTBI+ $(\mathrm{n}=11)$ individuals and TB patients $(\mathrm{n}=21)$ specific for $\mathrm{rGlcB}$ and rHspX (C); *: statistical significance compared to CS individuals; **: statistical significance compared to RA LTBI- individuals; ***: statistical significance compared to CS-LTBI+ individuals. The mean \pm standard errors of the mean is presented. 
TST are also based on cellular immune responses, like IGRAs or flow cytometry, but their general use remains controversial mainly due to their higher cost (Matulis et al. 2008, Nienhaus et al. 2013).

In this study, we initially investigated the expansion of PB Th1-specific cells of LTBI RA patients. Here, it was shown that Th1-specific cells expanded in the presence of recombinant Mtb antigens in RA LTBI+ patients, similar to CS-LTBI+ controls and TB patients. Other studies have also shown that the frequency of Th1 cells in the PB of RA patients was higher than in healthy controls (Chen et al. 2012) and that specific Th1 responses to ESAT- 6 and CFP-10 were similar to the responses observed in IGRA (Vassilopoulos et al. 2008). However, it is important to note that $30 \%$ of $\mathrm{RA} \mathrm{LTBI}+$ patients did not respond well to HspX. This fact reinforces the difficulty in diagnosing LTBI in RA individuals, as reported elsewhere (Matulis et al. 2008, Barry et al. 2009, Tannus-Silva et al. 2012).

Th17 cells from RA patients and CS did not expand in the presence of antigens, independent of the TST status. In an elegant study, Torrado and Cooper (2010) proposed that the Th17 cell population driven by the innate immune response is the first Th phenotype in TB granulomas. Thus, our results prompted us to hypothesise that Th17 cells would be a better surrogate marker for active TB once active TB patients showed specific
Th17 induction (Khader et al. 2007). We also investigated whether there were differences in IL-6 levels between RA patients that correlated with their TB latency status. We observed that RA LTBI+ patients had higher plasma levels of IL-6 compared to RA LTBI- individuals. This increase in IL-6 levels may be associated with a lack of HspX-responding Th1 cells in our three RA LTBI+ individuals.

A balance between effector and regulatory mechanisms may determine the outcome of an infection and could be beneficial for both the host and the pathogen in some cases. Additionally, Treg cells constitute a key component of peripheral tolerance by suppressing potentially autoreactive $\mathrm{T}$ cells and preventing autoimmune diseases. Recent reports have indicated that Treg cells may also contribute to the suppression of anti-TB immune responses by transient overexpression of TGF- $\beta$ and IL-10 (Ribeiro-Rodrigues et al. 2006, Chen et al. 2007, Ellner 2010). These results imply that pathogenspecific Treg cells can prevent infection-induced pathology, but may also prolong the persistence of the pathogen by suppressing protective immune responses.

There is a consensus that $5-10 \%$ of individuals with LTBI could develop active disease during their life span. When these individuals have risk factors such as previous contact with active TB patients or compromised immune systems, this percentage increases significantly.
A RA LTBI-

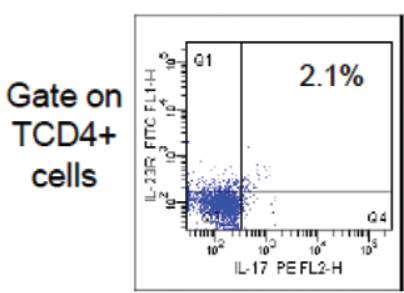

RA LTBI+

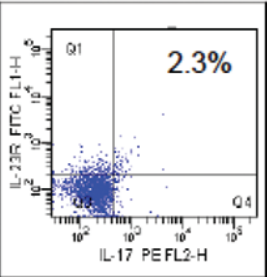

CS

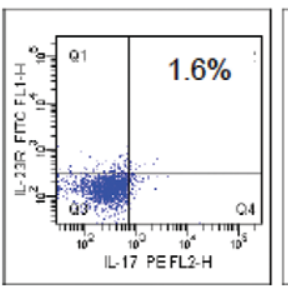

CS-LTBI+

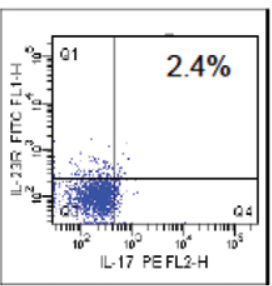

TB

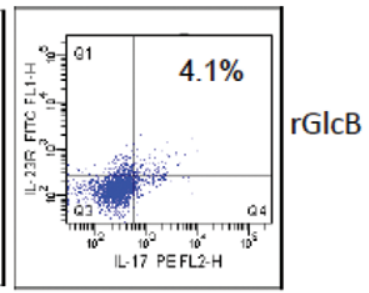

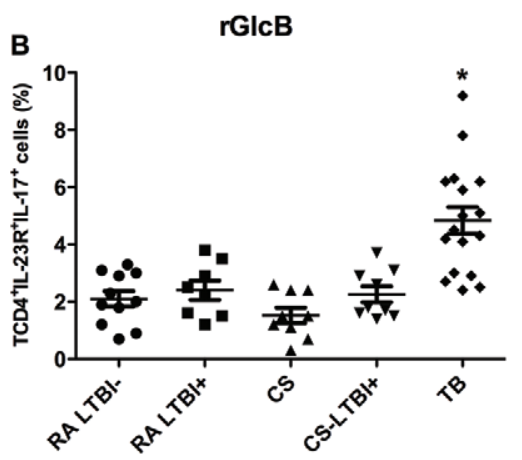

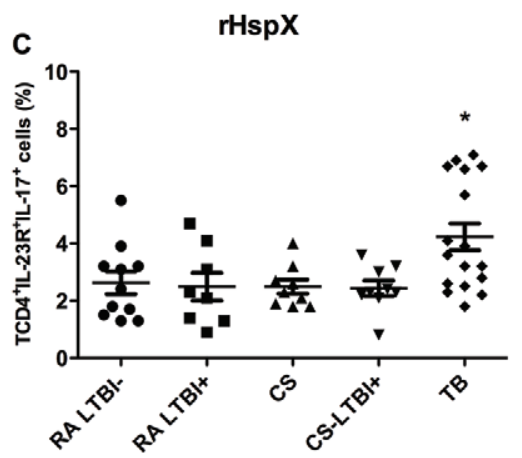

D

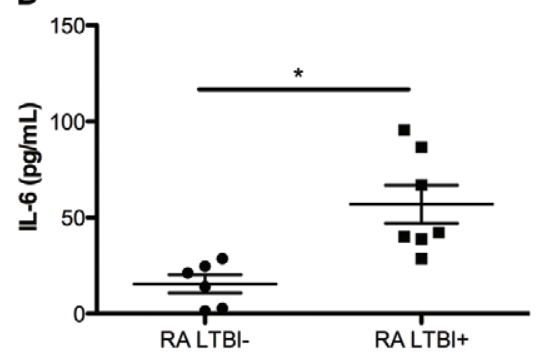

Fig. 2: evaluation of specific T-helper (Th) 17 [CD4 ${ }^{+}$interleukin (IL)-23R+IL-17A+] immune response to recombinant malate synthase (rGlcB) or recombinant heat shock protein $\mathrm{X}(\mathrm{rHspX})$ in peripheral blood mononuclear cells (PBMCs) cultures of rheumatoid arthritis (RA) with latent tuberculosis infection (RA-LTBI+), RA LTBI+, control subjects (CS), CS-LTBI+ individuals and TB patients. A: dot plots representing specific Th17 immune response in RA LTBI+, RA LTBI-, CS, CS-LTBI+ individuals and TB patients. Percentage of Th17 cells among RA LTBI+ $(\mathrm{n}=$ 10), RA LTBI- $(\mathrm{n}=11), \mathrm{CS}(\mathrm{n}=12)$, CS-LTBI+ $(\mathrm{n}=11)$ individuals and TB patients $(\mathrm{n}=21)$ specific for $\mathrm{rGlcB}(\mathrm{B})$ and $\mathrm{rHspX}(\mathrm{C})$; D: levels of IL-6 among RA LTBI+ $(n=7)$ and RA LTBI- $(n=6)$ patients measured by ELISA and expressed in pg/mL. Asterisk means: $p<0.05$. The mean \pm standard errors of the mean is presented. PBMCs cultured in vitro for $96 \mathrm{~h}$ were analysed by flow cytometry. 
Therefore, the presence of RA is a risk factor for the development of active $\mathrm{TB}$, especially in individuals with latent infection. Here, we show that within a high-risk population, $14.3 \%$ of RA patients progress to active TB disease. The Th cell subset responses studied in RA patients after the development of active TB were similar to the responses observed in TB patients. The Mtb-specific T cell populations increased with TB development; thus, Mtb GlcB or HspX-specific Th1, Th17 and Treg cells are induced during TB activation. These results imply that these Th cell subsets may participate in the progression from latent to active TB disease despite the presence of an autoimmune disease.

Although the authors acknowledge the small number of enrolled patients, the number of RA patients recruited and that voluntarily participated in the study correspond to approximately $20 \%$ of patients at this outpatient clinic at the Clinical Hospital, a government hospital. Thus, we believe that this limitation did not influence the outcome of our analysis.
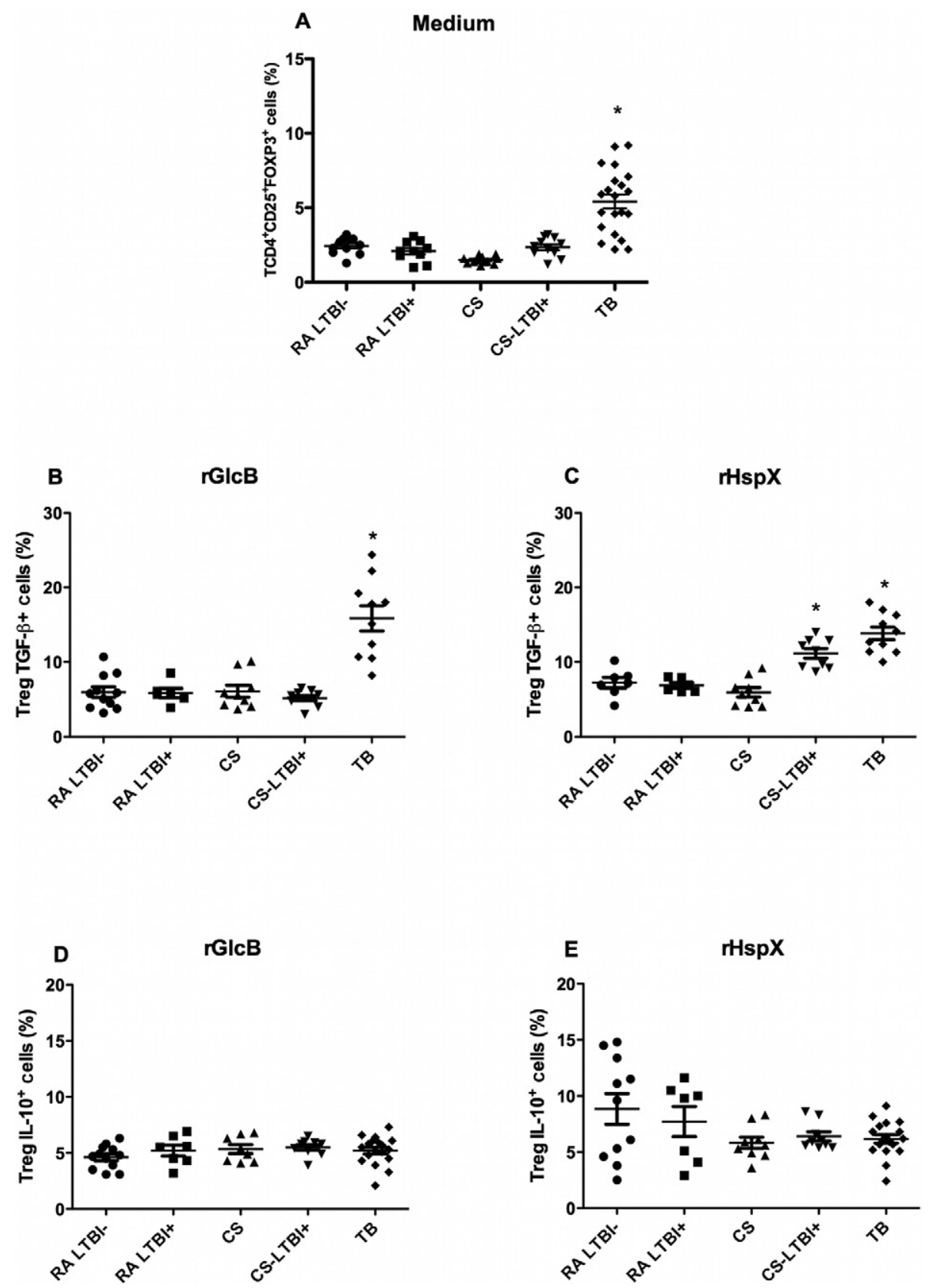

Fig. 3: percentage of regulatory T cells among peripheral blood of rheumatoid arthritis (RA) with latent tuberculosis infection (RA-LTBI+), RA LTBI-, control subjects (CS), CS-LTBI+ individuals and TB patients (A). Peripheral blood mononuclear cells were cultivated in vitro for $96 \mathrm{~h}$ with recombinant malate synthase $(\mathrm{rGlcB})(\mathrm{B}$ and $\mathrm{D})$ or with recombinant heat shock protein $\mathrm{X}(\mathrm{rHspX})(\mathrm{C}$ and $\mathrm{E})$ and analysed by flow cytometry. Percentages of Treg transforming growth factor (TGF)- $\beta+$ cells are shown in B, C. D, E: percentages of Treg interleukin (IL)-10+ cells; FOXP3: forkhead box protein 3. Asterisk means: $\mathrm{p}<0.05$. The mean \pm standard errors of the mean is presented. 

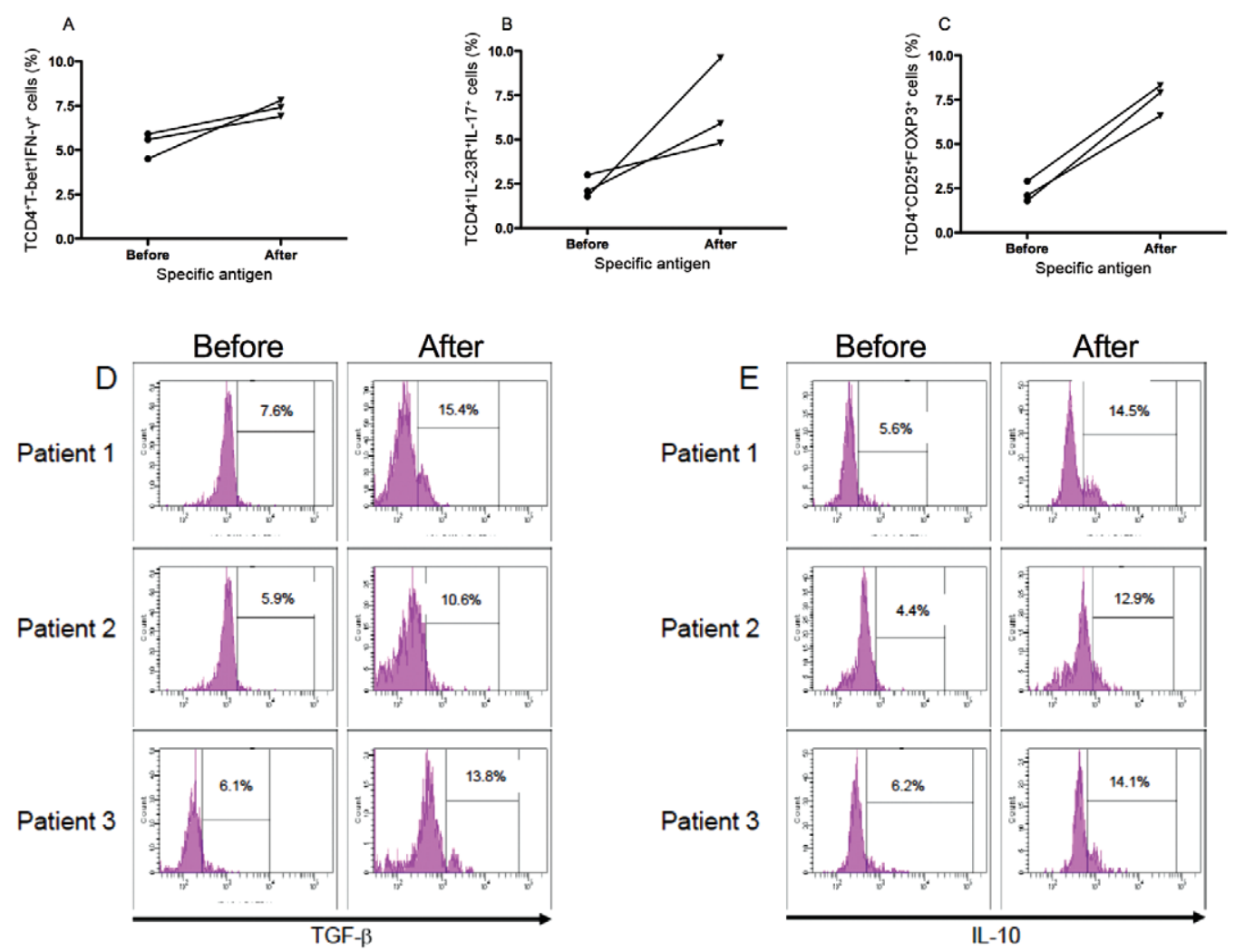

Fig. 4: tuberculosis activation induces increase of specific T-helper (Th)1 (A), Th17 (B), Treg (C), Treg transforming growth factor (TGF)- $\beta+(D)$ and Treg interleukin (IL)-10+ (E) cells in rheumatoid arthritis (RA) patients. Peripheral blood mononuclear cells were cultured in vitro for $96 \mathrm{~h}$ with medium, phytohaemagglutinin (PHA), recombinant malate synthase ( $\mathrm{rGlcB}$ ) or recombinant heat shock protein X (rHspX) and analysed by flow cytometry. The results shown were obtained after stimulation with $\mathrm{rHspX}$ antigen. The mean \pm standard errors of the mean is presented. FOXP3: forkhead box protein 3; IFN: interferon. Asterisk means: $\mathrm{p}<0.05$.

In conclusion, LTBI RA patients had Th1-specific immune responses to $\mathrm{rGlcB}$ and $\mathrm{rHspX}$. These results demonstrate that the rGlcB and $\mathrm{rHspX}$ can be used to formulate new tests to identify LTBI in RA patients.

\section{ACKNOWLEDGEMENTS}

To the flow cytometry core facilities of ACCG and Sarah Nogueira, for comments.

\section{REFERENCES}

Aaltonen KJ, Virkki LM, Malmivaara A, Konttinen YT, Nordstrom DC, Blom M 2012. Systematic review and meta-analysis of the efficacy and safety of existing TNF blocking agents in treatment of rheumatoid arthritis. PLoS ONE 7: e30275.

Araújo-Filho JA, Vasconcelos ACJ, Sousa EM, Kipnis A, JunqueiraKipnis AP 2008. Cellular responses to MPT-51, GlcB and ESAT-6 among MDR-TB and active tuberculosis patients in Brazil. Tuberculosis 88: 474-481.

Babu S, Bhat SQ, Kumar NP, Kumaraswami V, Nutman T B 2010. Regulatory T cells modulate Th17 responses in patients with positive tuberculin skin test results. J Infect Dis 201: 20-31.

Barry CE, Boshoff HI, Dartois V, Dick T, Ehrt S, Flynn J, Schnappingers D, Wilkinson RJ, Young D 2009. The spectrum of latent tuberculosis: rethinking the biology and intervention strategies. Nat Rev Microbiol 7: 845-855.
Boissier MC, Assier E, Falgarone G, Bessis N 2008. Shifting the imbalance from Th1/Th2 to Th17/Treg: the changing rheumatoid arthritis paradigm. Joint Bone Spine 75: 373-375.

Brennan FM, McInnes IB 2008. Evidence that cytokines play a role in rheumatoid arthritis. J Clin Invest 118: 3537-3545.

Chabaud M, Durand JM, Buchs N, Fossiez F, Page G, Frappart L, Miossec P 1999. Human interleukin-17: a T cell-derived proinflammatory cytokine produced by the rheumatoid synovium. Arthritis Rheum 42: 963-970.

Chen J, Li J, Gao H, Wang C, Luo J, Lv Z, Li X 2012. Comprehensive evaluation of different T-helper cells subsets differentiation and function in rheumatoid arthritis. J Biomed Biotechnol 2012: ID 535361 .

Chen X, Zhou B, Li M, Deng Q, Wu X, Le X, Wu C, Larmonier N, Zhang $\mathrm{W}$, Zhang $\mathrm{H}$, Wang $\mathrm{H}$, Katsanis E 2007. $\mathrm{CD}^{+} \mathrm{CD}^{+} 5^{+} \mathrm{FOXP}^{+}$regulatory T cells suppress Mycobacterium tuberculosis immunity in patients with active disease. Clin Immunol 123: 50-59.

Cope AP 2008. T cells in rheumatoid arthritis. Arthritis Res Ther 10: $1-10$.

Cruz A, Khader SA, Torrado E, Fraga A, Pearl JE, Pedrosa J, Cooper AM, Castro AG 2009. CE: IFN-g regulates the induction and expansion of IL-17 producing CD4 T cells during mycobacterial infection. J Immunol 177: 1416-1420.

Ellner JJ 2010. Immunoregulation in TB: observations and implications. Clin Transl Sci 3: 23-28. 
Fontenot JD, Gavin MA, Rundensky AY 2003. FOXP3 programs the development and function of $\mathrm{CD} 4{ }^{+} \mathrm{CD} 25^{+}$regulatory cells. Nat Immunol 4: 330-336.

Guyot-Revol V, John AI, Hackfort S, Hinks T, Lalvani A 2006. Regulatory $\mathrm{T}$ cells are expanded in blood and disease sites in patients with tuberculosis. Am J Respir Crit Care Med 173: 803-810.

Hougardy JM, Place S, Hildebrand M, Drowart A, Debrie AS, Locht C, Marcart F 2007. Regulatory T cells depress immune response to protective antigens in active tuberculosis. Am J Respir Crit Care Med 176: 409-416.

Jick SS, Lieberman ES, Rahman MU, Choi HK 2006. Glucocorticoid use, other associated factors and the risk of tuberculosis. Arthritis Reum 55: 19-26.

Khader SA, Bell GK, Pearl JE, Fountain JJ, Rangel-Moreno J, Cilley G E, Shen F, Eaton S M, Gaffen SL, Swain SL, Locksley RM, Haynes L, Randall TD, Cooper AM 2007. IL-23 and IL-17 in the establishment of protective pulmonary $\mathrm{CD}^{+} \mathrm{T}$ cell responses after vaccination and during Mycobacterium tuberculosis challenge. Nat Immunol 8: 369-377.

Matulis G, Juni P, Villiger PM, Gadola SD 2008. Detection of latent tuberculosis in immunosuppressed patients with autoimmune diseases: performance of a Mycobacterium tuberculosis antigenspecific interferon gamma assay. Ann Rheum Dis 67: 84-90.

Mota LMH, Cruz BA, Brenol CV, Pereira IA, Rezende-Fronza LS, Bertolo MB, Freitas MVC, Silva NA, Louzada-Júnior P, Giorgi RDN, Lima RAC, Pinheiro GRC 2012. Consenso 2012 da Sociedade Brasileira de Reumatologia para o tratamento da artrite reumatóide. Rev Bras Reumatol 52: 135-174.

Mus AM, Cornelissen F, Asmawidjaja PS, Hamburg JP, Boon L, Hendriks RW, Lubberts E 2010. Interleukin-23 promotes Th17 differentiation by inhibiting T-bet and FOXP3 and is required for elevation of interleukin-22, but not interleukin-21, in autoimmune experimental arthritis. Arthritis Rheum 62: 1043-1050.

Nienhaus A, Ringshausen FC, Costa JT, Schablon A, Dominique Tripodi D 2013. IFN- $\gamma$ release assay versus tuberculin skin test for monitoring TB infection in health care workers. Expert Rev Anti Infect Ther 11: 37-48.

Okada M, Kita Y, Kanamaru N, Hashimoto S, Uchiyama Y, Mihara M, Inoue Y, Ohsugi Y, Kishimoto T, Sakatani M 2011. Anti-IL-6 receptor antibody causes less promotion of tuberculosis infection than anti-TNF- $\alpha$ antibody in mice. Clinical Dev Immunol 2011: e404929.

Rabahi MF, Junqueira-Kipnis AP, Dos Reis MC, Oelemann W, Conde MB 2007. Humoral response to HspX and GlcB to previous and recent infection by Mycobacterium tuberculosis. BMC Infect Dis 7: 148-151.

Reis MC, Rabahi MF, Kipnis A, Junqueira-Kipnis AP 2009. Health care workers humoral immune response against GLcB, MPT51 and HSPX from Mycobacterium tuberculosis. Braz J Infect Dis 13: $417-421$
Ribeiro-Rodrigues, R, Resende TC, Rojas R, Toosi Z, Dietze R, Boom WH, Maciel E, Hirsch CS 2006. A role of $\mathrm{CD}^{+} \mathrm{CD} 25^{+} \mathrm{T}$ cells in regulation of the immune response during human tuberculosis. Clin Exp Immunol 144: 25-34.

Roberts T, Beyers N, Aguirre A, Walzl G 2007. Immunosuppression during active tuberculosis is characterized by decreased interferon- $\gamma$ production and $\mathrm{CD} 25$ expression with elevated forkhead box P3, transforming growth factor- $\beta$ and interleukin- 4 mRNA levels. J Infect Dis 195: 870-878.

Rueda CM, Marín ND, García LF, Rojas M 2010. Characterization of $\mathrm{CD} 4$ and $\mathrm{CD} 8 \mathrm{~T}$ cells producing IFN- $\gamma$ in human latent and active tuberculosis. Tuberculosis 90: 346-353.

Scott DL, Wolfe F, Huizinga TW 2010. Rheumatoid arthritis. Lancet 376: 1094-1108.

Scriba TJ, Kalsdorf B, Abrahams D, Isaacs F, Hofmeister J, Black G, Hassan HY, Wilkinson RJ, Walzl G, Gerderbloem SJ, Mahomed H, Hussey GD, Hanekom WA 2008. Distinct, specific IL-17 and IL-22-producing $\mathrm{CD}^{+} \mathrm{T}$ cells subsets contribute to the human anti-mycobacterial immune response. J Immunol 180: 1962-1970.

Tannus-Silva DS, Silva BDS, Torres PP, Santana PJJ, JunqueiraKipnis AP, Rabahi MF 2012. Latent tuberculosis in rheumatoid arthritis: evaluating cellular response and high-resolution computed tomography. Arch Bronconeumol 48: 144-149.

Tavares RCO, Salgado J, Moreira VB, Ferreira MAS, Mello FCQ, Leung JAW Leung JAW, Singh M, Fonseca LS, Saad MHF 2006. Cell proliferation and interferon- $\gamma$ response to recombinant MBP-3, NarL, MT-10.3 and 16kDa Mycobacterium tuberculosis antigens in Brazilian tuberculosis patients. Mem Inst Oswaldo Cruz 101: 857-861.

Torrado E, Cooper AM 2010. IL-17 and Th17 cells in tuberculosis. Cytokine Growth Factor Rev 21: 455-462.

Vassilopoulos D, Stamoulis N, Hadziyannis E, Archimandritis AJ 2008. Usefulness of enzyme-linked immunospot assay (Elispot) compared to tuberculin skin testing for latent tuberculosis screening in rheumatic patients scheduled for anti-tumor necrosis factor treatment. $J$ Rheumatol 35: 1271-1276.

Wang W, Shao S, Jiao Z, Guo M, Xu H, Wang S 2012. The Th17/Treg imbalance and cytokine environment in peripheral blood of patients with rheumatoid arthritis. Rheumatol Int 4: 887-893.

WHO - World Health Organization 2003. Risk of TB infection among HCWs in the era of HIV and MDR-TB. Available from: who.int/ tb/events/archive/icmeeting_oct07/02_pai.pdf.

WHO - World Health Organization 2011. Fact sheets in tuberculosis: Regional Office for the Western Pacific. Available from: wpro. who.int/media_centre/fact_sheets/fs_20090327.htm.

Zhang L, Li Y, Li Y, Qi L, Liu X, Yuan C, Hu N, Ma D, Li Z, Yang Q, Li W, Li J 2012. Increased frequencies of Th22 cells as well as Th17 cells in the peripheral blood of patients with ankylosing spondylitis and rheumatoid arthritis. PLOS ONE 7: e31000. 